\title{
Effect of Different Extrusion Processing Parameters on Physical Properties of Soy White Flakes and High Protein Distillers Dried Grains-Based Extruded Aquafeeds
}

\author{
Sushil K. Singh ${ }^{1} \&$ K. Muthukumarappan ${ }^{1}$ \\ ${ }^{1}$ Department of Agricultural \& Biosystems Engineering, South Dakota State University, Brookings, SD, USA \\ Correspondence: Sushil K. Singh, Department of Agricultural and Biosystems Engineering, South Dakota State \\ University, Brookings, SD, USA. E-mail: sushil.singh@sdstate.edu
}

Received: February 11, 2014

Accepted: August 13, 2014 Online Published: August 18, 2014

doi:10.5539/jfr.v3n6p107

URL: http://dx.doi.org/10.5539/jfr.v3n6p107

\begin{abstract}
Nutritionally balanced ingredient blends for catla (Catla catla), belonging to the family Cyprinidae, were extruded using single screw extruder. The extrusion was carried out at five levels of soy white flakes content $(21 \%, 29 \%, 40 \%, 52 \%$, and $59 \% \mathrm{db})$, five levels of moisture content $(15,19,25,31$, and $35 \% \mathrm{db})$ and five levels of barrel temperature $\left(100,110,125,140\right.$, and $150{ }^{\circ} \mathrm{C}$ ) using three different die nozzles (having L/D ratios 3.33, 5.83 , and 7.25). Blends with net protein content of $32.5 \%$ contains soy white flakes, along with high protein distillers dried grains (HP-DDG), corn flour, corn gluten meal, fish meal, vitamin, and mineral mix. A central composite rotatable design (CCRD) and response surface methodology (RSM) was used to investigate the significance of independent and interaction effects of the extrusion process variables on the extrudates physical properties namely pellet durability index, bulk density, water absorption and solubility indices and expansion ratio. Quadratic polynomial regression equations were developed to correlate the product responses and process variables as well as to obtain the response surfaces plots. The independent variables had significant $(P<0.05)$ effects on physical properties of extrudates: (i) higher soy white flakes content increased the pellet durability index and water absorption index, but decreased the water solubility index, (ii) higher temperature decreased pellet durability index, bulk density and water solubility index, (iii) increased L/D ratio from 3.33 to 7.25 increased the pellet durability index, expansion ratio but decreased the bulk density of the extrudates.
\end{abstract}

Keywords: bulk density, die, extrusion, pellet durability index, unit density, soy white flakes

\section{Introduction}

In the food producing industries, aquaculture is one of the fastest growing sectors (FAO, 2012) and plays a pivotal role for the maintenance of commercial fishery markets (O'Mahoney et al., 2011). In aquaculture, diet is often the single largest operating cost item and can represent over $50 \%$ of the operating costs in intensive aquaculture (El-Sayed, 1999, 2004). Protein is the most important nutrient of the fish feed. The main protein source used in aquafeed production is primarily fish meal which is supplied through the consumption of wild fish stocks. Indubitably, with the increasing rate of farmed fish production (FAO, 2008) and consequently rising prices of fishmeal (Hardy, 2010), the continued use of fishmeal as the main protein source of the feed will no longer be ecologically and economically sustainable in the long run. Therefore, aquaculture industry now is focusing on alternative protein sources such as plant proteins as inexpensive source of protein to minimize production cost.

Soy white flakes and High Protein - Distiller Dried Grains (HP-DDG) contain significant amount of protein and are thus a possible alternative source of protein for aquaculture feeds (Chin et al., 1989; Wu et al., 1994, 1996). Use of soy products like full fatted soybean meal, defatted toasted soybean meal (SBM) and defatted untoasted soybean meal or soy white flakes is becoming common (Fallahi et al., 2012). Romarheim et al. (2005) found that extrusion of soy white flakes diet increased the digestibility of protein and all amino acids compared to the unextruded soy white flakes diet probably due to the reduction in trypsin inhibitor activity. Dersjant-Li, (2002) reported that soy protein isolate can be used to replace $40-100 \%$ fish meal without negative impact on growth performance of shrimp. Distillers Dried Grains (DDG) and Distillers Dried Grains with Solubles (DDGS), a co-product from corn-based dry grind fuel ethanol manufacturing, is also a viable protein source. Research 
carried out by Wu et al. $(1994,1996 a)$ indicated that tilapia fish can be grown with DDGS, and can improve the economic viability of aquaculture farms.

Extrusion cooking is widely used in the food and feed industries because of versatility during processing and the ability to produce various final textural properties (Mercier et al., 1989). Extruded aquafeed are designed to floater sink based on the fish species requirement. One of the important quality parameters for fish feed is floatability (Bandyopadhyay \& Ranjan 2001; Rolfe et al., 2001) which depends on the unit density of extrudates. During extrusion cooking, the extent of expansion affects the unit density of the extrudates. Expansion can be monitored by changing the nature and type of ingredients used and the extruder process parameters. In the food industry, puffed products are often produced by using starch based ingredients, while texturized products are often produced by using protein based ingredients (Kokini et al., 1992a, 1992b). Extrudate properties of starch based products depend on the extent of gelatinization occurring inside the extruder barrel. The formation of elastic melt inside the barrel depends on the extent of gelatinization. (Case et al., 1992; Lin et al., 2000; Ilo et al., 1996; Ibanoglu et al., 1996; Sokhey et al., 1994). Expansion occurs due to the flashing of water vapor when the elastic melt exits through the die nozzle. (Lam \& Flores, 2003; Alves et al., 1999). On the other hand, ingredients with higher protein content shows limited degree of expansion due to plastic melt formation and protein denaturation inside the extruder barrel. The material is in plastic and homogeneous state and when it exits through the die nozzle there is a sudden pressure drop resulting in the formation of voids. Due to this void formation the final product becomes more porous and fibrous textured (Singh et al., 1991; Gwiazda et al., 1987; Sandra \& Jose, 1993). Depending on the type of species, aquaculture feed requires 26 to $50 \%$ protein content. (Lovell, 1988).

Extrusion process depends on many factors which includes the pressure developed inside the die and the degree to which the screw is filled. These variables in combination with the type and composition of raw ingredients used, affects operational capabilities (Mercier et al., 1989). Extruder die too have an impact on the processing conditions. For example, in case of circular dies, nozzle dimensions (i.e., nozzle diameter and length) will affect process conditions and performance (Chinnaswamy et al., 1987).

The objective of thisstudy was to examine the effect of varying level of soy white flakesas the fish meal replacer, barrel temperature, die aspect ratio, and moisture content on physical properties of soy white flakesand HP-DDG based extrudates.

\section{Materials and Methods}

\subsection{Blends Preparation}

Five isocaloric $(302 \mathrm{kcal} / 100 \mathrm{~g})$ different blends were adjusted to a target protein content of $\sim 32.5 \% \mathrm{db}$ and a target fat content of $\sim 3.5 \%$. The total energy content for each blend was determined based on the fraction of protein, lipid and carbohydrate contributing to the dietary energy. The total energy content was calculated based on the energy content of fractions namely, $4.5 \mathrm{kcal} / \mathrm{g}$ for protein, $9.1 \mathrm{kcal} / \mathrm{g}$ for lipid and $4.1 \mathrm{kcal} / \mathrm{g}$ for carbohydrate. The ingredient components of the feed blends are provided in Table 1. Soy white flakes was kindly donated by South Dakota Soybean Processors, Volga, SD. HP-DDG was obtained from the Dakota Ethanol LLC (Wentworth, SD). Corn gluten meal and fishmeal were purchased from Consumer Supply Distributing Co. (Sioux City, IA). Corn flour was from Cargill Dry Ingredients (Paris, IL). Vitamin and mineral premix was obtained from Lortscher Agri Service, Inc. (Bern, Kansas, USA). Soybean oil was provided from USDA (Brookings, SD). The different ingredients were mixed in a laboratory model Hobart mixer (Hobart Corporation, Troy, Ohio, USA) for 10 minutes; the moisture content of the ingredient mix was adjusted by adding required quantities of water during mixing. The resulting blends were then stored at ambient temperature overnight until processing. 
Table 1. Ingredient composition of feed blends

\begin{tabular}{llllll}
\hline Ingredients & Blend 1 & Blend 2 & Blend 3 & Blend 4 & Blend 5 \\
\hline Soy white flakes & 21 & 29 & 40 & 52 & 59 \\
HP-DDG & 39 & 32 & 20 & 9 & 1 \\
Corn gluten meal & 2 & 2 & 2 & 2 & 2 \\
Corn flour & 30 & 30 & 30 & 30 & 30 \\
Fish meal & 5 & 5 & 5 & 5 & 5 \\
Soybean oil & 1 & 1 & 1 & 1 & 1 \\
Vitamin \& mineral mix & 2 & 2 & 2 & 2 & 2 \\
Total & $\mathbf{1 0 0}$ & $\mathbf{1 0 0}$ & $\mathbf{1 0 0}$ & $\mathbf{1 0 0}$ & $\mathbf{1 0 0}$ \\
\hline
\end{tabular}

\subsection{Extrusion Processing}

Extrusion experiments were performed using a single screw extruder (BrabenderPlasti-Corder, Model PL 2000, South Hackensack, NJ), which was powered by a $7.5 \mathrm{hp}$ motor with an operating range of screw speeds from 0-210 rpm. The extruder had a barrel length-to-diameter ratio of 20:1 and a barrel diameter of $19 \mathrm{~mm}$. A uniform $19.05 \mathrm{~mm}$ pitch screw with compression ratio of 3:1 was used in the experiments (Figure 1). The screw had a variable flute depth, with a depth at the feed portion of $19.05 \mathrm{~mm}$, and near the die of $3.81 \mathrm{~mm}$. The raw materials were fed manually to the extruder in constant quantities. Experiments were conducted using five levels of soy white flakes $(21,29,40,52$, and $59 \% \mathrm{db})$, five levels of temperature gradient in the barrel $\left(45-100-100{ }^{\circ} \mathrm{C}\right.$, $45-110-110^{\circ} \mathrm{C}, 45-125-125^{\circ} \mathrm{C}, 45-140-140^{\circ} \mathrm{C}$, and $45-150-150^{\circ} \mathrm{C}$ ) hereafter referred as temperature of 100,110 , 125,140 , and $150^{\circ} \mathrm{C}$, and five levels of moisture content $(15,19,25,31$, and $35 \% \mathrm{db})$, for three different die nozzles with various L/D ratios $(3.33,5.83$ and 7.25$)$ (Table 2). During the experiment the screw speed of extruder was maintained at $150 \mathrm{rpm}$.

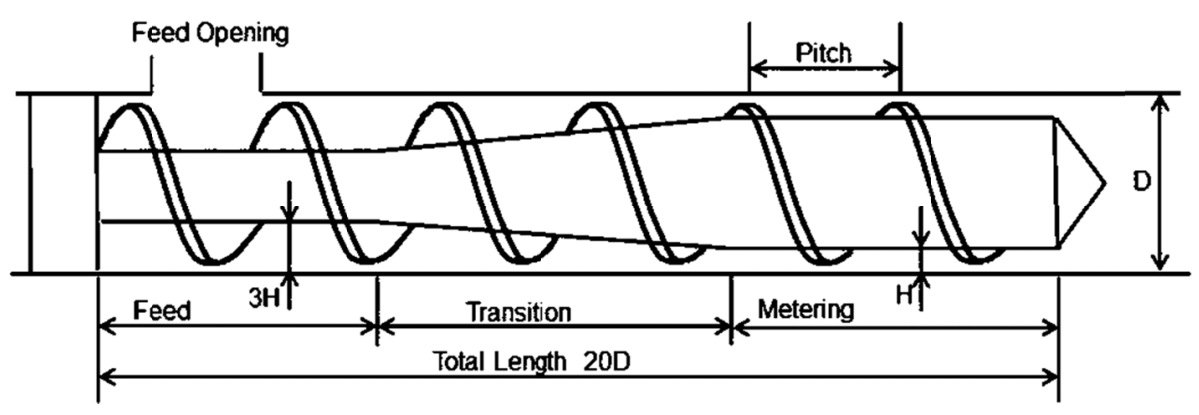

Figure 1. Schematic representation of screw in a single screw extruder

Table 2. Dimensions of die used in this study

\begin{tabular}{llll}
\hline Die No. & $\begin{array}{l}\text { Diameter of nozzle } \\
(\mathbf{m m})\end{array}$ & $\begin{array}{l}\text { Length of nozzle } \\
(\mathbf{m m})\end{array}$ & $\begin{array}{l}\text { L/D ratio } \\
(-)\end{array}$ \\
\hline D1 & 6 & 20.0 & 3.33 \\
D2 & 3 & 17.5 & 5.83 \\
D3 & 2 & 14.5 & 7.25 \\
\hline
\end{tabular}

\subsection{Experimental Design and Statistical Analysis}

In the present study, a central composite rotatable design (CCRD) was used to evaluate the effect of soy white flakes, moisture content, temperature and L/D of die nozzle on the physical properties of the extrudate. Pellet durability index, bulk density, water absorption and solubility indices and expansion ratio of the extrudates were measured as the response/dependent variables. The measurements were completed in triplicate, except for expansion ratio, which were measured with ten replications. The collected data were then analyzed with Proc GLM procedure to determine the treatment combination effects using SAS v9.3 (SAS Institute, Cary, NC). Then post hoc LSD tests were used to determine where the specific differences occurred. The experimental design was 
developed using Design-Expert 8.0.7.1 (Statease, Minneapolis, MN), which consisted of 3 numerical independent variables of soy white flakes $\left(X_{1}\right)$, moisture content $\left(X_{2}\right)$ and $\mathrm{T}\left(X_{3}\right)$ each at five levels and one categorical variable of die nozzle configuration $\left(X_{4}\right)$ at three levels. The experimental design points (in coded and actual values) are shown in Table 3.Using Equation 1, the numerical independent variables in actual form $\left(X_{1}, X_{2}\right)$ were converted to their coded form $\left(x_{1}, x_{2}\right)$.

$$
x_{i}=\frac{\left(X_{i}-X_{0}\right)}{\Delta X}
$$

Where $x_{\mathrm{i}}$ is the dimensionless coded value of the $\mathrm{i}^{\text {th }}$ independent variable, and $X i, X_{0}$, and $\Delta X$ correspond to the actual value, actual value at the center point, and the step change of the $i^{\text {th }}$ variable, respectively.

Table 3. Independent numerical and categorical variables and their levels

\begin{tabular}{lllllll}
\hline Numerical variables & Symbol & \multicolumn{5}{c}{ Coded variable levels } \\
\hline & & -1.682 & -1 & 0 & 1 & 1.682 \\
\cline { 3 - 6 } Soy white flakes (\%) & $X_{1}$ & 21 & 29 & 40 & 52 & 59 \\
Moisture content (\% db) & $X_{2}$ & 15 & 19 & 25 & 31 & 35 \\
Temperature $\left({ }^{\circ} \mathrm{C}\right)$ & $X_{3}$ & 100 & 110 & 125 & 140 & 150 \\
Categorical variable & & D1 & D2 & D3 & & \\
\cline { 3 - 5 } L/D (-) & $X_{4}[1]$ & 1 & 0 & -1 & \\
& $X_{4}[2]$ & 0 & 1 & -1 & \\
\hline
\end{tabular}

For each categorical variable, 20 experiments were performed in randomized order including six replications at the design center to obtain an accurate estimation of the experimental error (Table 4a and $4 \mathrm{~b}$ ). The pellet durability index $\left(Y_{P D I}\right)$, bulk density $\left(Y_{B D}\right)$, water absorption index $\left(Y_{W A I}\right)$, water solubility index $\left(Y_{W S I}\right)$ and expansion ratio $\left(Y_{E R}\right)$ were taken as the five responses of the designed experiments. The quadratic polynomial equation was used to describe the effect of the independent variables in terms of linear, quadratic and their interactions on the dependent variables as given by Equation 2 .

$$
Y_{i}=b_{0}+\sum_{i=1}^{4} b_{i} X_{i}+\sum_{i=1}^{4} b_{i i} X_{i}^{2}+\sum_{i=1}^{3} \sum_{j=i+1}^{4} b_{i j} X_{i} X_{j}+\varepsilon
$$

Where $Y_{i}$ is the predicted response; $b_{0}$ is the interception coefficient; $b_{i}, b_{i i}$, and $b_{i j}$ are coefficients of the linear, quadratic, and interaction terms; $\varepsilon$ is the random error; and $X_{i}$ is the independent variables studied. The fitness of the model was evaluated and the interactions between the independent and dependent variables were identified by using an analysis of variance (ANOVA) presented in Tables 5 and 6.The goodness of fit of the second order equation was expressed by the coefficient of determination $\left(R^{2}\right)$ and its statistical significance was determined by $F$-test (Table 7). 3D response surfaces were used to visualize interactive effects of the independent variables.

\subsection{Measurement of Physical Properties}

\subsubsection{Pellet Durability Index}

Approximately $100 \mathrm{~g}$ of extrudates from each blend were manually sieved (U.S.A. standard testing, ASTM E-11 specification, Daigger, Vernon Hills, IL) to remove initial fines, and then tumbled in a pellet durability tester (Model PDT-110, Seedburo Equipment Company, Chicago, IL) for $10 \mathrm{~min}$. Afterwards, the samples were again sieved, and then weighed on an electronic balance (Explorer Pro, Model: EP4102, Ohaus, Pine Brook, NJ) (ASAE, 2004). Pellet durability index was calculated following the Equation 3:

$$
\text { Pellet durability index }(\%)=\left(\frac{\mathrm{M}_{\mathrm{a}}}{\mathrm{M}_{\mathrm{b}}} \times 100\right)
$$

where, $\mathrm{M}_{\mathrm{a}}$ was the mass (g) after tumbling and $\mathrm{M}_{\mathrm{b}}$ was the sample mass (g) before tumbling.

\subsubsection{Bulk Density}

Bulk density was determined as the ratio of the mass of extrudates that they filled up to a given bulk volume and measured using a standard bushel tester (Seedburo Equipment Company, Chicago, IL) following the method recommended by USDA (2009).

\subsubsection{Water Absorption Index and Water Solubility Index}

Extrudates were ground to fine powders using a coffee grinder (Black \& Decker ${ }^{\circledR}$ Corporation, Towson, ML, 
USA). The ground extrudates $(2.5 \mathrm{~g})$ was suspended in distilled water $(30 \mathrm{~mL})$ in a tarred $60 \mathrm{~mL}$ centrifuge tube. The suspension was stirred intermittently and centrifuged at $3000 \mathrm{~g}$ for $10 \mathrm{~min}$. The supernatant was decanted into a tarred aluminums cup and dried at $135^{\circ} \mathrm{C}$ for $2 \mathrm{~h}$ (AACC, 2000). The weight of the gel remaining in the centrifuge tube was measured. The water absorption index and water solubility index were calculated by Equation 4 and 5, respectively:

$$
\text { Water absorption index (unitless) }=\left(\frac{\mathrm{w}_{\mathrm{g}}}{\mathrm{w}_{\mathrm{ds}}}\right)
$$

where $\mathrm{W}_{\mathrm{g}}$ is the weight of gel $(\mathrm{g})$, and $\mathrm{W}_{\mathrm{ds}}$ is the weight of dry sample $(\mathrm{g})$.

$$
\text { Water solubility index }(\%)=\left(\frac{\mathrm{W}_{\mathrm{ss}}}{\mathrm{W}_{\mathrm{ds}}} \times 100\right)
$$

where $\mathrm{W}_{\mathrm{ss}}$ is the weight of dry solids of supernatant $(\mathrm{g})$, and $\mathrm{W}_{\mathrm{ds}}$ is the weight of dry sample $(\mathrm{g})$.

\subsubsection{Expansion Ratio}

The radial expansion ratio of the extrudates was measured as the ratio of the diameter of the extrudates to the diameter of the die orifice.

\begin{tabular}{|c|c|c|c|c|c|c|c|c|c|}
\hline \multirow[t]{3}{*}{ Run } & \multicolumn{5}{|c|}{ Coded variables } & \multicolumn{4}{|c|}{ Actual variables } \\
\hline & \multirow[t]{2}{*}{$x_{1}$} & \multirow[t]{2}{*}{$x_{2}$} & \multirow[t]{2}{*}{$x_{3}$} & \multicolumn{2}{|c|}{$x_{4}$} & \multirow{2}{*}{$\begin{array}{l}X_{1} \\
(\%)\end{array}$} & \multirow{2}{*}{$\begin{array}{c}X_{2} \\
(\% \mathrm{db})\end{array}$} & \multirow{2}{*}{$\begin{array}{c}X_{3} \\
\left({ }^{\circ} \mathrm{C}\right)\end{array}$} & \multirow{2}{*}{$\begin{array}{l}X_{4} \\
(-)\end{array}$} \\
\hline & & & & $x_{4}[1]$ & $x_{4}[2]$ & & & & \\
\hline 1 & 0 & 0 & -1.682 & 1 & 0 & 40 & 25 & 100 & D1 \\
\hline 2 & 0 & 0 & 0 & 0 & 1 & 40 & 25 & 125 & D2 \\
\hline 3 & -1 & -1 & 1 & 1 & 0 & 29 & 19 & 140 & D1 \\
\hline 4 & 1.682 & 0 & 0 & 1 & 0 & 59 & 25 & 125 & D1 \\
\hline 5 & 1 & -1 & -1 & 0 & 1 & 52 & 19 & 110 & D2 \\
\hline 6 & 0 & 0 & 0 & 1 & 0 & 40 & 25 & 125 & D1 \\
\hline 7 & 1 & 1 & -1 & 0 & 1 & 52 & 31 & 110 & D2 \\
\hline 8 & 1 & -1 & 1 & 0 & 1 & 52 & 19 & 140 & D2 \\
\hline 9 & 0 & 0 & 0 & -1 & -1 & 40 & 25 & 125 & D3 \\
\hline 10 & 1 & -1 & 1 & 1 & 0 & 52 & 19 & 140 & D1 \\
\hline 11 & -1 & 1 & -1 & 0 & 1 & 29 & 31 & 110 & D2 \\
\hline 12 & 1 & 1 & 1 & 1 & 0 & 52 & 31 & 140 & D1 \\
\hline 13 & 0 & 0 & 1.682 & 0 & 1 & 40 & 25 & 150 & D2 \\
\hline 14 & -1 & -1 & -1 & 0 & 1 & 29 & 19 & 110 & D2 \\
\hline 15 & 0 & 0 & 0 & -1 & -1 & 40 & 25 & 125 & D3 \\
\hline 16 & 1 & -1 & 1 & -1 & -1 & 52 & 19 & 140 & D3 \\
\hline 17 & -1 & 1 & -1 & 1 & 0 & 29 & 31 & 110 & D1 \\
\hline 18 & 0 & 0 & 0 & -1 & -1 & 40 & 25 & 125 & D3 \\
\hline 19 & -1.682 & 0 & 0 & 1 & 0 & 21 & 25 & 125 & D1 \\
\hline 20 & -1 & -1 & -1 & -1 & -1 & 29 & 19 & 110 & D3 \\
\hline 21 & 0 & 0 & 0 & 1 & 0 & 40 & 25 & 125 & D1 \\
\hline 22 & 0 & -1.682 & 0 & 0 & 1 & 40 & 15 & 125 & D2 \\
\hline 23 & 0 & 0 & -1.682 & -1 & -1 & 40 & 25 & 100 & D3 \\
\hline 24 & -1 & -1 & -1 & 1 & 0 & 29 & 19 & 110 & D1 \\
\hline 25 & 1.682 & 0 & 0 & 0 & 1 & 59 & 25 & 125 & D2 \\
\hline 26 & 0 & 0 & 0 & -1 & -1 & 40 & 25 & 125 & D3 \\
\hline 27 & 1 & 1 & -1 & 1 & 0 & 52 & 31 & 110 & D1 \\
\hline 28 & 0 & 0 & 0 & -1 & -1 & 40 & 25 & 125 & D3 \\
\hline 29 & -1.682 & 0 & 0 & 0 & 1 & 21 & 25 & 125 & D2 \\
\hline 30 & 0 & 0 & 0 & 0 & 1 & 40 & 25 & 125 & D2 \\
\hline
\end{tabular}

Table 4a. Experimental design layout 
Table 4b. Experimental design layout

\begin{tabular}{|c|c|c|c|c|c|c|c|c|c|}
\hline \multirow[t]{3}{*}{ Run } & \multicolumn{5}{|c|}{ Coded variables } & \multicolumn{4}{|c|}{ Actual variables } \\
\hline & \multirow[t]{2}{*}{$x_{1}$} & \multirow[t]{2}{*}{$x_{2}$} & \multirow[t]{2}{*}{$x_{3}$} & \multicolumn{2}{|c|}{$x_{4}$} & \multirow{2}{*}{$\begin{array}{c}X_{1} \\
(\%)\end{array}$} & \multirow{2}{*}{$\frac{X_{2}}{(\% \mathrm{db})}$} & \multirow{2}{*}{$\begin{array}{c}X_{3} \\
\left({ }^{\circ} \mathrm{C}\right)\end{array}$} & \multirow{2}{*}{$\begin{array}{l}X_{4} \\
(-)\end{array}$} \\
\hline & & & & $x_{4}[1]$ & $x_{4}[2]$ & & & & \\
\hline 31 & 0 & 0 & 0 & 0 & 1 & 40 & 25 & 125 & D2 \\
\hline 32 & -1 & 1 & 1 & 0 & 1 & 29 & 31 & 140 & D2 \\
\hline 33 & 1 & -1 & -1 & 1 & 0 & 52 & 19 & 110 & D1 \\
\hline 34 & 0 & 0 & 0 & -1 & -1 & 40 & 25 & 125 & D3 \\
\hline 35 & 1 & 1 & -1 & -1 & -1 & 52 & 31 & 110 & D3 \\
\hline 36 & 0 & 0 & -1.682 & 0 & 1 & 40 & 25 & 100 & D2 \\
\hline 37 & 0 & 1.682 & 0 & 1 & 0 & 40 & 35 & 125 & D1 \\
\hline 38 & 0 & -1.682 & 0 & 1 & 0 & 40 & 15 & 125 & D1 \\
\hline 39 & 0 & 0 & 0 & 1 & 0 & 40 & 25 & 125 & D1 \\
\hline 40 & -1 & 1 & 1 & 1 & 0 & 29 & 31 & 140 & D1 \\
\hline 41 & 0 & 0 & 0 & 0 & 1 & 40 & 25 & 125 & D2 \\
\hline 42 & 0 & 1.682 & 0 & -1 & -1 & 40 & 35 & 125 & D3 \\
\hline 43 & 1.682 & 0 & 0 & -1 & -1 & 59 & 25 & 125 & D3 \\
\hline 44 & -1 & -1 & 1 & 0 & 1 & 29 & 19 & 140 & D2 \\
\hline 45 & 0 & 0 & 0 & 0 & 1 & 40 & 25 & 125 & D2 \\
\hline 46 & 0 & 0 & 0 & 0 & 1 & 40 & 25 & 125 & D2 \\
\hline 47 & 0 & 0 & 0 & 1 & 0 & 40 & 25 & 125 & D1 \\
\hline 48 & 1 & 1 & 1 & 0 & 1 & 52 & 31 & 140 & D2 \\
\hline 49 & 0 & 0 & 0 & 1 & 0 & 40 & 25 & 125 & D1 \\
\hline 50 & -1 & 1 & -1 & -1 & -1 & 29 & 31 & 110 & D3 \\
\hline 51 & -1.682 & 0 & 0 & -1 & -1 & 21 & 25 & 125 & D3 \\
\hline 52 & 0 & 0 & 1.682 & -1 & -1 & 40 & 25 & 150 & D3 \\
\hline 53 & 0 & 1.682 & 0 & 0 & 1 & 40 & 35 & 125 & D2 \\
\hline 54 & 0 & -1.682 & 0 & -1 & -1 & 40 & 15 & 125 & D3 \\
\hline 55 & 1 & 1 & 1 & -1 & -1 & 52 & 31 & 140 & D3 \\
\hline 56 & 0 & 0 & 0 & 1 & 0 & 40 & 25 & 125 & D1 \\
\hline 57 & 1 & -1 & -1 & -1 & -1 & 52 & 19 & 110 & D3 \\
\hline 58 & -1 & -1 & 1 & -1 & -1 & 29 & 19 & 140 & D3 \\
\hline 59 & -1 & 1 & 1 & -1 & -1 & 29 & 31 & 140 & D3 \\
\hline 60 & 0 & 0 & 1.682 & 1 & 0 & 40 & 25 & 150 & D1 \\
\hline
\end{tabular}


Table 5. Analysis of Variance (ANOVA) for pellet durability index and bulk density

\begin{tabular}{|c|c|c|c|c|c|c|c|c|c|}
\hline \multirow{2}{*}{ Source } & \multirow{2}{*}{ df } & \multicolumn{4}{|c|}{ Pellet durability index } & \multicolumn{4}{|c|}{ Bulk density } \\
\hline & & SS & MS & F value & $P$-value & SS & MS & F value & $P$-value \\
\hline Model & 17 & 702.94 & 41.35 & 6.41 & $<0.0001$ & $1.93 \times 10^{-1}$ & $1.13 \times 10^{-2}$ & 16.54 & $<0.0001$ \\
\hline$X_{1}$ & 1 & 77.83 & 77.83 & 12.06 & 0.0012 & $7.18 \times 10^{-4}$ & $7.18 \times 10^{-4}$ & 1.05 & 0.3117 \\
\hline$X_{2}$ & 1 & 17.18 & 17.18 & 2.66 & 0.1102 & $9.79 \times 10^{-3}$ & $9.79 \times 10^{-3}$ & 14.30 & 0.0005 \\
\hline$X_{3}$ & 1 & 203.67 & 203.67 & 31.56 & $<0.0001$ & $2.61 \times 10^{-2}$ & $2.61 \times 10^{-2}$ & 38.06 & $<0.0001$ \\
\hline$X_{4}$ & 2 & 198.72 & 99.36 & 15.40 & $<0.0001$ & $1.24 \times 10^{-1}$ & $6.19 \times 10^{-2}$ & 90.37 & $<0.0001$ \\
\hline$X_{I}^{2}$ & 1 & 0.58 & 0.58 & 0.09 & 0.7663 & $5.95 \times 10^{-6}$ & $5.95 \times 10^{-6}$ & 0.01 & 0.9262 \\
\hline$X_{2}^{2}$ & 1 & 105.47 & 105.47 & 16.35 & 0.0002 & $1.28 \times 10^{-2}$ & $1.28 \times 10^{-2}$ & 18.66 & $<0.0001$ \\
\hline$X_{3}{ }^{2}$ & 1 & 5.40 & 5.40 & 0.84 & 0.3654 & $4.65 \times 10^{-5}$ & $4.65 \times 10^{-5}$ & 0.07 & 0.7956 \\
\hline$X_{1} X_{2}$ & 1 & 10.60 & 10.60 & 1.64 & 0.2071 & $5.00 \times 10^{-4}$ & $5.00 \times 10^{-4}$ & 0.73 & 0.3979 \\
\hline$X_{1} X_{3}$ & 1 & 1.64 & 1.64 & 0.25 & 0.6169 & $1.23 \times 10^{-5}$ & $1.23 \times 10^{-5}$ & 0.02 & 0.8939 \\
\hline$X_{1} X_{4}$ & 2 & 32.82 & 16.41 & 2.54 & 0.0907 & $5.14 \times 10^{-3}$ & $2.57 \times 10^{-3}$ & 3.75 & 0.0316 \\
\hline$X_{2} X_{3}$ & 1 & 1.55 & 1.55 & 0.24 & 0.6270 & $2.96 \times 10^{-4}$ & $2.96 \times 10^{-4}$ & 0.43 & 0.5146 \\
\hline$X_{2} X_{4}$ & 2 & 7.67 & 3.84 & 0.59 & 0.5565 & $6.50 \times 10^{-4}$ & $3.25 \times 10^{-4}$ & 0.47 & 0.6254 \\
\hline$X_{3} X_{4}$ & 2 & 40.41 & 20.20 & 3.13 & 0.0540 & $1.27 \times 10^{-2}$ & $6.36 \times 10^{-5}$ & 9.29 & 0.0005 \\
\hline Residual & 42 & 271.01 & 6.45 & - & - & $2.88 \times 10^{-2}$ & $6.85 \times 10^{-4}$ & - & - \\
\hline Lack of fit & 27 & 202.96 & 7.52 & 1.66 & 0.1536 & $2.37 \times 10^{-2}$ & $8.76 \times 10^{-4}$ & 2.58 & 0.0291 \\
\hline Pure error & 15 & 68.05 & 4.54 & - & - & $5.10 \times 10^{-3}$ & $3.40 \times 10^{-4}$ & - & - \\
\hline
\end{tabular}

$+\mathrm{df}-$ degree of freedom, $\mathrm{SS}-\mathrm{Sum}$ of squares, MS - Mean square.

Table 6. Analysis of Variance (ANOVA) for water solubility index and expansion ratio

\begin{tabular}{|c|c|c|c|c|c|c|c|c|c|}
\hline \multirow{2}{*}{ Source } & \multirow{2}{*}{ df } & \multicolumn{4}{|c|}{ Water solubility index } & \multicolumn{4}{|c|}{ Expansion ratio } \\
\hline & & SS & MS & F value & $P$-value & $\mathbf{S S}$ & MS & F value & $P$-value \\
\hline Model & 17 & 22.25 & 1.31 & 2.77 & 0.0037 & $4.20 \times 10^{-1}$ & $2.50 \times 10^{-2}$ & 13.22 & $<0.0001$ \\
\hline$X_{I}$ & 1 & 2.91 & 2.91 & 6.15 & 0.0172 & $3.38 \times 10^{-3}$ & $3.38 \times 10^{-3}$ & 1.82 & 0.1842 \\
\hline$X_{2}$ & 1 & 3.64 & 3.64 & 7.71 & 0.0082 & $2.20 \times 10^{-2}$ & $2.20 \times 10^{-2}$ & 11.65 & 0.0014 \\
\hline$X_{3}$ & 1 & 6.22 & 6.22 & 13.17 & 0.0008 & $2.50 \times 10^{-2}$ & $2.50 \times 10^{-2}$ & 13.71 & 0.0006 \\
\hline$X_{4}$ & 2 & 0.25 & 0.12 & 0.26 & 0.7700 & $2.30 \times 10^{-1}$ & $1.10 \times 10^{-1}$ & 61.92 & $<0.0001$ \\
\hline$X_{1}^{2}$ & 1 & 6.12 & 6.12 & 12.95 & 0.0008 & $5.14 \times 10^{-3}$ & $5.14 \times 10^{-3}$ & 2.77 & 0.1035 \\
\hline$X_{2}^{2}$ & 1 & 1.27 & 1.27 & 2.68 & 0.1090 & $3.10 \times 10^{-2}$ & $3.10 \times 10^{-2}$ & 16.76 & 0.0002 \\
\hline$X_{3}{ }^{2}$ & 1 & 0.19 & 0.19 & 0.41 & 0.5277 & $2.20 \times 10^{-2}$ & $2.20 \times 10^{-2}$ & 11.94 & 0.0013 \\
\hline$X_{1} X_{2}$ & 1 & 0.21 & 0.21 & 0.45 & 0.5055 & $5.12 \times 10^{-3}$ & $5.12 \times 10^{-3}$ & 2.76 & 0.1039 \\
\hline$X_{1} X_{3}$ & 1 & 0.61 & 0.61 & 1.30 & 0.2604 & $8.62 \times 10^{-4}$ & $8.62 \times 10^{-4}$ & 0.46 & 0.4991 \\
\hline$X_{1} X_{4}$ & 2 & 0.05 & 0.03 & 0.06 & 0.9452 & $1.10 \times 10^{-2}$ & $5.74 \times 10^{-3}$ & 3.10 & 0.0557 \\
\hline$X_{2} X_{3}$ & 1 & 0.00 & 0.00 & 0.00 & 0.9927 & $5.55 \times 10^{-4}$ & $5.55 \times 10^{-4}$ & 0.30 & 0.5871 \\
\hline$X_{2} X_{4}$ & 2 & 1.35 & 0.68 & 1.43 & 0.2501 & $3.13 \times 10^{-3}$ & $1.57 \times 10^{-3}$ & 0.84 & 0.4371 \\
\hline$X_{3} X_{4}$ & 2 & 0.08 & 0.04 & 0.09 & 0.9160 & $6.50 \times 10^{-2}$ & $3.30 \times 10^{-2}$ & 17.58 & $<0.0001$ \\
\hline Residual & 42 & 19.84 & 0.47 & - & - & $7.80 \times 10^{-2}$ & $1.85 \times 10^{-3}$ & - & - \\
\hline Lack of fit & 27 & 15.87 & 0.59 & 2.22 & 0.0541 & $6.60 \times 10^{-2}$ & $2.45 \times 10^{-5}$ & 3.16 & 0.0114 \\
\hline Pure error & 15 & 3.97 & 0.26 & - & - & $1.20 \times 10^{-2}$ & $7.76 \times 10^{-4}$ & - & - \\
\hline
\end{tabular}

${ }^{+} \mathrm{df}-$ degree of freedom, SS - Sum of squares, MS - Mean square. 
Table 7. Final equation in terms of coded factors after excluding the insignificant terms for pellet durability index, bulk density, water solubility index and expansion ratio

\begin{tabular}{|c|c|c|c|c|}
\hline Coded model equations & $R^{2}$ & $\operatorname{Adj} R^{2}$ & $\operatorname{Pred} R^{2}$ & Adeq. precision \\
\hline$Y_{P D I}=+89.91+1.38 x_{1}-2.23 x_{3}-1.86 x_{4}[1]-0.61 x_{4}[2]+1.56 x_{2}^{2}$ & 0.72 & 0.61 & 0.36 & 9.98 \\
\hline$Y_{B D}=+0.42-0.015 x_{2}-0.025 x_{3}+0.058 x_{4}[1]-4.89 \times 10^{-3} x_{4}$ & & & & \\
\hline $\begin{array}{c}+1.92 \times 10^{-3} x_{1} x_{4}[1]+0.013 x_{1} x_{4}[2]+0.02 x_{3} x_{4}[1] \\
+2.87 \times 10^{-3} x_{3} x_{4}[2]+0.017 x_{2}^{2}\end{array}$ & 0.87 & 0.82 & 0.69 & 19.03 \\
\hline$Y_{W S I}=+14.07-0.27 x_{1}-0.30 x_{2}-0.39 x_{3}+0.38 x_{1}^{2}$ & 0.53 & 0.34 & -0.09 & 6.52 \\
\hline $\begin{aligned} Y_{E R}= & +1.19-0.023 x_{2}+0.025 x_{3}-0.081 x_{4}[1]+0.013 x_{4}[2] \\
& -0.056 x_{3} x_{4}[1]+0.023 x_{3} x_{4}[2]-0.027 x_{2}^{2}-0.023 x_{3}^{2}\end{aligned}$ & 0.84 & 0.78 & 0.62 & 14.85 \\
\hline
\end{tabular}

\section{Results and Discussion}

Response surface methodology (RSM) was used to analyze the relationship between the dependent and independent variables. The predictive models in coded terms (i.e., $Y_{P D I}, Y_{B D}, Y_{W S I}$ and $Y_{E R}$ ) are presented in Table 7. On the contrary, the final equations in actual form are defined for each type of categorical factor separately. The final equations in actual form obtained for pellet durability index $\left(Y_{P D I}\right)$, bulk density $\left(Y_{B D}\right)$, water solubility index $\left(Y_{W S I}\right)$ and expansion ratio $\left(Y_{E R}\right)$ for each level of categoric variable (D1, D2 and D3) are given in Table 8.

Table 8. Best-fit response surface models for extrudate physical properties

\begin{tabular}{|c|c|c|c|c|}
\hline L/D & Response Surface Model & $R^{2}$ & $\begin{array}{l}\text { Std. } \\
\text { Deviation }\end{array}$ & $\begin{array}{l}F \\
\text { Statistic }\end{array}$ \\
\hline \multirow[t]{4}{*}{ D1 } & $\begin{array}{l}Y_{P D I}=+155.64+0.10 X_{1}-3.14 X_{2}-0.46 X_{3}+0.01 X_{1} X_{2}-1.51 \times 10^{-3} X_{1} X_{3}+ \\
2.94 \times 10^{-3} X_{2} X_{3}-8.74 \times 10^{-4} X_{1}^{2}+0.05 X_{2}^{2}+1.57 \times 10^{-3} X_{3}^{2}\end{array}$ & 0.72 & 2.54 & 6.41 \\
\hline & $\begin{array}{l}Y_{B D}=+0.81+1.51 \times 10^{-3} X_{1}-0.02 X_{2}-6.48 \times 10^{-4} X_{3}-6.90 \times 10^{-5} X_{I} X_{2}+4.15 \times 10^{-6} X_{I} X_{3} \\
-4.07 \times 10^{-5} X_{2} X_{3}+2.80 \times 10^{-6} X_{1}^{2}+5.20 \times 10^{-4} X_{2}^{2}+4.61 \times 10^{-6} X_{3}^{2}\end{array}$ & 0.87 & 0.026 & 16.54 \\
\hline & $\begin{array}{l}Y_{\text {WSI }}=+30.07-0.17 X_{I^{-}} 0.41 X_{2}-0.07 X_{3}+1.42 \times 10^{-3} X_{I} X_{2}-9.28 \times 10^{-4} X_{I} X_{3}- \\
1.50 \times 10^{-5} X_{2} X_{3}+2.84 \times 10^{-3} X_{1}^{2}+5.18 \times 10^{-3} X_{2}^{2}+2.96 \times 10^{-4} X_{3}^{2}\end{array}$ & 0.53 & 0.69 & 2.77 \\
\hline & $\begin{array}{l}Y_{E R}=-0.97+0.02 X_{1}+0.04 X_{2}+0.02 X_{3}-2.21 \times 10^{-4} X_{1} X_{2}-3.47 \times 10^{-5} X_{1} X_{3}+ \\
5.58 \times 10^{-5} X_{2} X_{3}-8.24 \times 10^{-5} X_{1}^{2}-8.11 \times 10^{-4} X_{2}^{2}-1.01 \times 10^{-4} X_{3}^{2}\end{array}$ & 0.84 & 0.043 & 13.22 \\
\hline \multirow[t]{4}{*}{ D2 } & $\begin{array}{l}Y_{P D I}=+172.09+0.23 X_{1}-3.29 X_{2}-0.59 X_{3}+0.01 X_{1} X_{2}-1.51 \times 10^{-3} X_{I} X_{3}+ \\
2.94 \times 10^{-3} X_{2} X_{3}-8.74 \times 10^{-4} X_{I}^{2}+0.05 X_{2}^{2}+1.57 \times 10^{-3} X_{3}^{2}\end{array}$ & 0.72 & 2.54 & 6.41 \\
\hline & $\begin{array}{l}Y_{B D}=+0.82+2.45 \times 10^{-3} X_{1}-0.02 X_{2}-1.80 \times 10^{-3} X_{3}-6.90 \times 10^{-5} X_{I} X_{2}+ \\
4.15 \times 10^{-6} X_{1} X_{3}-4.07 \times 10^{-5} X_{2} X_{3}+2.80 \times 10^{-6} X_{1}^{2}+5.20 \times 10^{-4} X_{2}^{2}+4.61 \times 10^{-6} X_{3}^{2}\end{array}$ & 0.87 & 0.026 & 16.54 \\
\hline & $\begin{array}{l}Y_{W S I}=+27.68-0.17 X_{I^{-}} 0.33 X_{2}-0.06 X_{3}+1.42 \times 10^{-3} X_{I} X_{2}-9.28 \times 10^{-4} X_{1} X_{3}- \\
1.50 \times 10^{-5} X_{2} X_{3}+2.84 \times 10^{-3} X_{1}^{2}+5.18 \times 10^{-3} X_{2}^{2}+2.96 \times 10^{-4} X_{3}^{2}\end{array}$ & 0.53 & 0.69 & 2.77 \\
\hline & $\begin{array}{l}Y_{E R}=-1.56+0.02 X_{1}+0.04 X_{2}+0.03 X_{3^{-}} 2.21 \times 10^{-4} X_{I} X_{2}-3.47 \times 10^{-5} X_{I} X_{3}+ \\
5.58 \times 10^{-5} X_{2} X_{3}-8.24 \times 10^{-5} X_{1}^{2}-8.11 \times 10^{-4} X_{2}^{2}-1.01 \times 10^{-4} X_{3}^{2}\end{array}$ & 0.84 & 0.043 & 13.22 \\
\hline \multirow[t]{4}{*}{ D3 } & $\begin{array}{l}Y_{P D I}=+184.83+0.05 X_{1}-3.31 X_{2}-0.61 X_{3}+0.01 X_{1} X_{2}-1.51 \times 10^{-3} X_{1} X_{3}+ \\
2.94 \times 10^{-3} X_{2} X_{3}-8.74 \times 10^{-4} X_{1}^{2}+0.05 X_{2}^{2}+1.57 \times 10^{-3} X_{3}^{2}\end{array}$ & 0.72 & 2.54 & 6.41 \\
\hline & $\begin{array}{l}Y_{B D}=+1.09+7.74 \times 10^{-5} X_{1}-0.02 X_{2}-3.51 \times 10^{-3} X_{3^{-}} 6.90 \times 10^{-5} X_{I} X_{2}+4.15 \times 10^{-6} X_{I} X_{3^{-}} \\
4.07 \times 10^{-5} X_{2} X_{3}+2.80 \times 10^{-6} X_{1}^{2}+5.20 \times 10^{-4} X_{2}^{2}+4.61 \times 10^{-6} X_{3}^{2}\end{array}$ & 0.87 & 0.026 & 16.54 \\
\hline & $\begin{array}{l}Y_{W S I}=+28.31-0.17 X_{1}-0.36 X_{2^{-}} 0.06 X_{3}+1.42 \times 10^{-3} X_{1} X_{2}-9.28 \times 10^{-4} X_{1} X_{3}- \\
1.50 \times 10^{-5} X_{2} X_{3}+2.84 \times 10^{-3} X_{1}^{2}+5.18 \times 10^{-3} X_{2}^{2}+2.96 \times 10^{-4} X_{3}^{2}\end{array}$ & 0.53 & 0.69 & 2.77 \\
\hline & $\begin{array}{l}Y_{E R}=-1.62+0.02 X_{1}+0.04 X_{2}+0.03 X_{3}-2.21 \times 10^{-4} X_{1} X_{2}-3.47 \times 10^{-5} X_{1} X_{3}+ \\
5.58 \times 10^{-5} X_{2} X_{3}-8.24 \times 10^{-5} X_{1}^{2}-8.11 \times 10^{-4} X_{2}^{2}-1.01 \times 10^{-4} X_{3}^{2}\end{array}$ & 0.84 & 0.043 & 13.22 \\
\hline
\end{tabular}


Overall, changing the level oftemperature content significantly affected $(P<0.05)$ all the resulting physical properties. Changing the level of moisture content significantly affected $(P<0.05)$ all the resulting physical properties except pellet durability index. Whereas changing soy white flakes content significantly affected $(P<0.05)$ pellet durability index, water absorption and solubility indices (Table 5 and 6$)$. The behavior observed for the treatment combinations were produced due to the various competing interaction effects (Table 9).

\subsection{Pellet Durability Index}

The response surface plot presented (Figure 2) showed that for all the L/D ratio, the pellet durability index of extrudates increased on increasing soy white flakes content and decreasing temperature. ANOVA showed that moisture content had no significant effect on pellet durability index and hence response surface plots of interaction effect involving moisture content are not shown. Lack of fit was not significant relative to the pure error, which meant the model was well fitted. The regression equation for pellet durability index in coded and actual form is shown in Table 7 and 8, respectively.

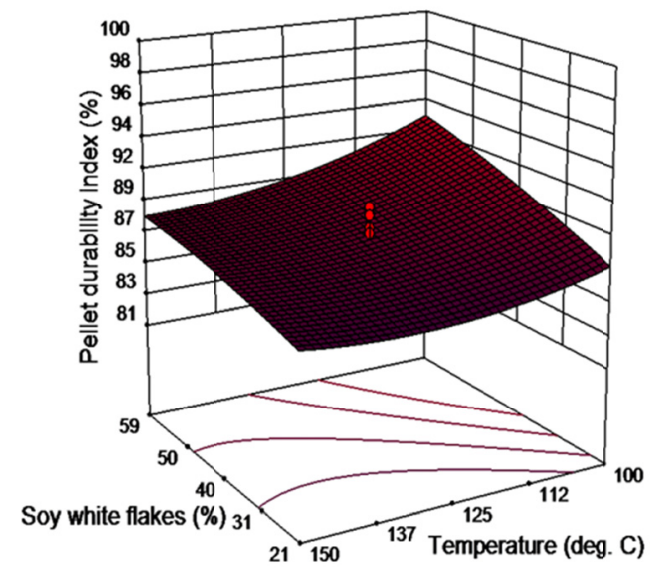

(A)

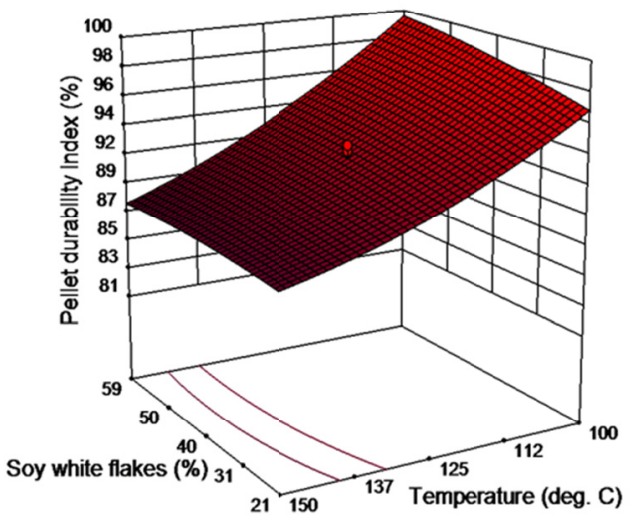

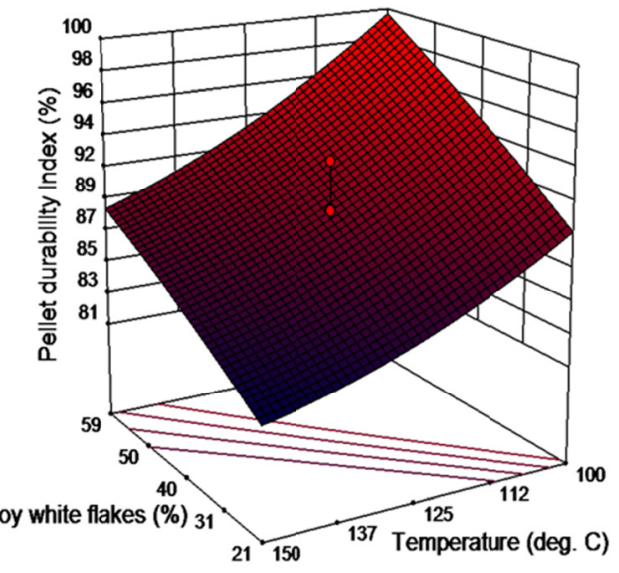

(B)

(C)

Figure 2. Response surface plots of pellet durability index for the effect of soy white flakes content and temperature at $25 \% \mathrm{db}$ moisture content at different die aspect ratio (L/D), (A) 3.33, (B) 5.83, and (C) 7.25

The values of pellet durability index of extruded products under experimental conditions are presented in Table 9 . The maximum and minimum pellet durability index values were related to the treatments with $40 \%$ soy white flakes, $25 \%$ moisture content, $125^{\circ} \mathrm{C}$ barrel temperature, $3.33 \mathrm{~L} / \mathrm{D}$ and $21 \%$ soy white flakes, $25 \%$ moisture content $125^{\circ} \mathrm{C}$ barrel temperature, $5.83 \mathrm{~L} / \mathrm{D}$ ratio, respectively (Table 9).

\subsection{Bulk Density}

The response surface plot presented (Figure 3) showed that for all the L/D ratio, the bulk density of extrudates decreased with an increasing of moisture content and temperature. A significant decrease in bulk density was observed when length to diameter ratio of die was increased from 3.33 to 7.25. ANOVA showed that soy white 
flakes had no significant effect on bulk density and hence response surface plots of interaction effect involving soy white flakes are not shown. The regression equation for bulk density in coded and actual form is shown in Table 7 and 8, respectively.
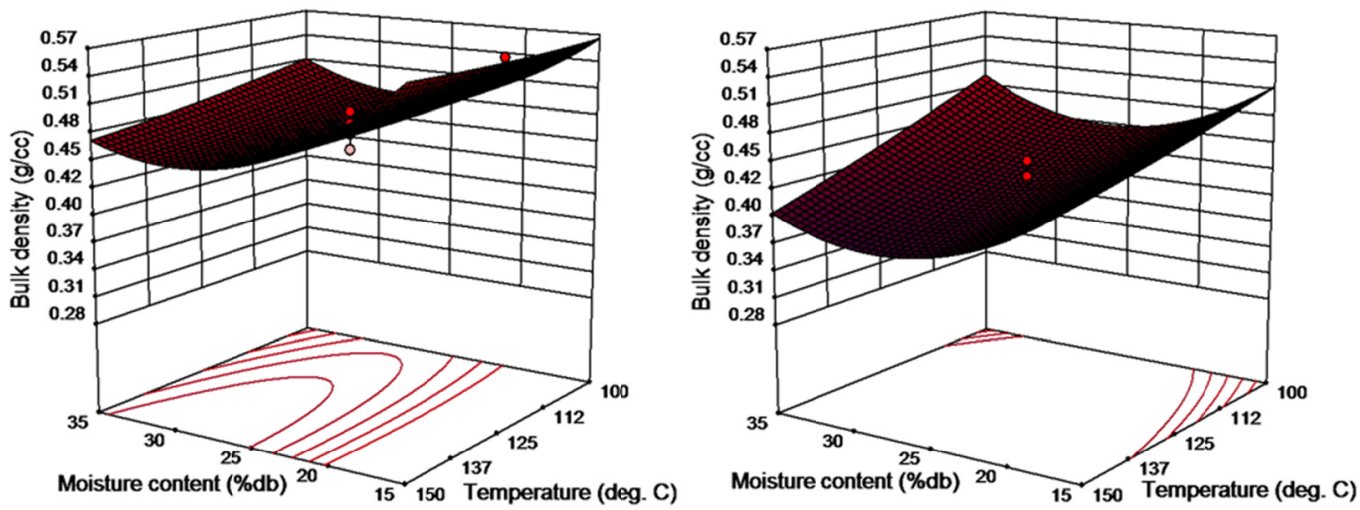

(A)

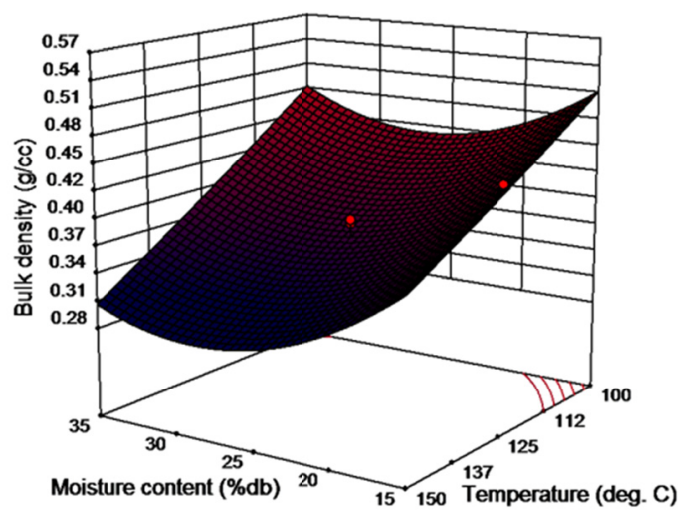

(B)

(C)

Figure 3. Response surface plots of bulk density for the effect of temperature and moisture content at $40 \% \mathrm{db}$ soy white flakes at different die aspect ratio (L/D), (A) 3.33, (B) 5.83, and (C) 7.25

The treatment combination effects of soy white flakes, moisture content, temperature and L/D ratio on bulk density of extrudates are presented in Table 9 . The lowest bulk density, $0.32 \mathrm{~g} / \mathrm{cm}^{3}$, was recorded at $29 \%$ soy white flakes, temperature of $140{ }^{\circ} \mathrm{C}$, moisture content of $31 \%, \mathrm{~L} / \mathrm{D}$ of 7.25 and at $59 \%$ soy white flakes, temperature of $125{ }^{\circ} \mathrm{C}$, moisture content of $25 \%, \mathrm{~L} / \mathrm{D}$ ratio of 7.25 ; and the highest bulk density of $0.57 \mathrm{~g} / \mathrm{cm}^{3}$, was obtained at $40 \%$ soy white flakes, temperature of $125^{\circ} \mathrm{C}$, moisture content of $15 \%$, and L/D of 3.33 (Table 9).

\subsection{Water Absorption Index}

Soy white flakes, moisture content and temperature significantly affected the water absorption index through a linear model (response surface plots were prepared but not shown due to linear model and to reduce the manuscript size). ANOVA analysis demonstrated that the linear model was significant $(P<0.05)$. Increasing the soy white flakes content from $21 \%$ to $52 \%$, there was a significant increase of in water absorption index. Water absorption index was found to increase when temperature was raised from $100{ }^{\circ} \mathrm{C}$ to $150^{\circ} \mathrm{C}$. With extruded corn grits, a similar trend was observed by Anderson et al. (1969). Furthermore, water absorption index also increased when moisture content level of the feed was increased from $15 \%$ to $35 \%$. This was in agreement with Williams et al. (1977) who observed that higher temperature and drier conditions could result in higher dextrinization, which could lead to a decreased extrudate water absorption index and higher extrudate water solubility index. According to Mason and Hoseney (1986), water absorption index indicates the part of the starch that was not affected by the extrusion cooking and maintained its internal structure. The experimental values of water absorption index of extrudates under different designed extrusion conditions are presented in Table 9. 


\subsection{Water Solubility Index}

Changing the level of soy white flakes, moisture content of ingredient mix and barrel temperature had significant effect $(P<0.05)$ on water solubility index (Table 6). Williams et al. (1977) reported that there was an inverse relationship between the water absorption index and water solubility index values of the extrudates. Similarly, in this study the water solubility index at $15 \%$ moisture content was higher compared to the water solubility index at $35 \%$ moisture content. Increasing the barrel temperature from 100 to $150{ }^{\circ} \mathrm{C}$ resulted in decrease in water solubility index. In another study, Chevanan et al. (2007) reported that there was no significant change in water solubility index of the DDGS-based extrudates due to the change in extruder barrel temperature. Increasing the soy white flakes content initially led to a significant decrease in water solubility index and then further increase in soy white flakes level resulted in slight increase in water solubility index. An inverse relationship between the water solubility index and water absorption index values were observed. This observation was in agreement with what Anderson et al. (1969), Williams et al. (1977), and Fallahi et al. (2012, 2013) reported.

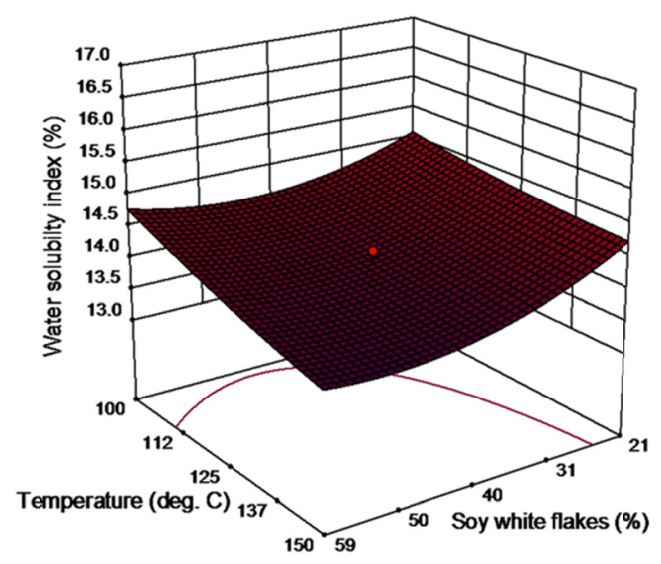

(A)

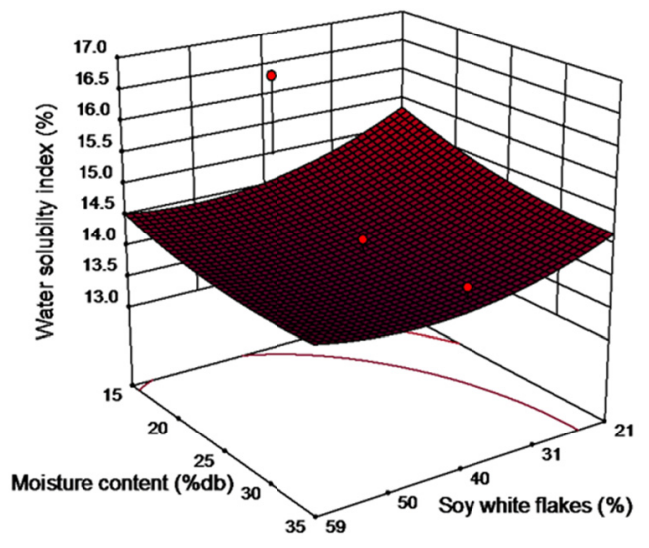

(C)

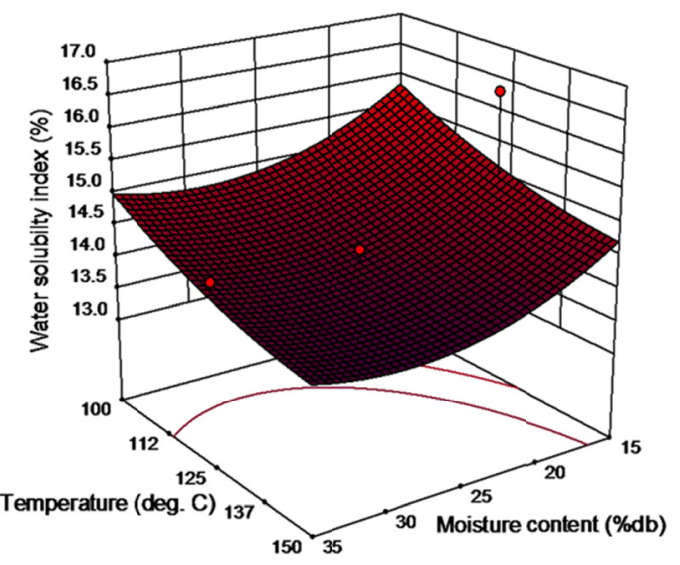

(B)

Figure 4. Response surface plots of water solubilityindexat die aspect ratio (L/D) of 7.25 for the effect of (A) temperature and soy white flakes content, (B) temperature and moisture content and (C) moisture and soy white flakes content

ANOVA analysis showed that quadratic effect of soy white flakes significantly affected water solubility index and all linear effects including soy white flakes, moisture content and temperature had significant effect on water solubility index. The interaction effect of soy white flakes, moisture content and temperature at L/D ratio at 7.25 on water solubility index was maximum and since L/D ratio does not significantly affect the water solubility index, the response surface plots of water solubility index at L/D ratio 7.25 are shown (Figure 4). Water solubility index is found to increase when temperature and soy white flakes content, temperature and moisture content, and moisture content and soy white flakes content are decreased (Figure 4). Lack of fit was not significant relative to the pure error, which meant the model was well fitted. The regression equation for the 
empirical relationship between water solubility index and the independent extrusion processing variables for each $\mathrm{L} / \mathrm{D}$ ratio is shown in Table 8 .

\subsection{Expansion Ratio}

The extent of puffing of extruded products is indicated by the expansion ratio. The response surface plot presented in Figure 5 showed that for all the L/D ratio, the expansion ratio of extrudates increased with an increasing moisture content and after reaching a maximum the expansion ratio decreased with further increasing in moisture content. The expansion ratio of extrudates increased for L/D ratio 5.83 and 7.25 but decreased for L/D ratio 3.33 with increase in temperature. ANOVA showed that soy white flakes had no significant effect on expansion ratio and hence response surface plots of interaction effect involving soy white flakes are not shown. The regression equation for the relationship between expansion ratio and independent variables in terms of coded and actual form for each L/D ratio is shown in Table 7 and 8 , respectively. Increasing the level of moisture content of ingredient mix and temperature had a significant effect on expansion ratio of extrudates.
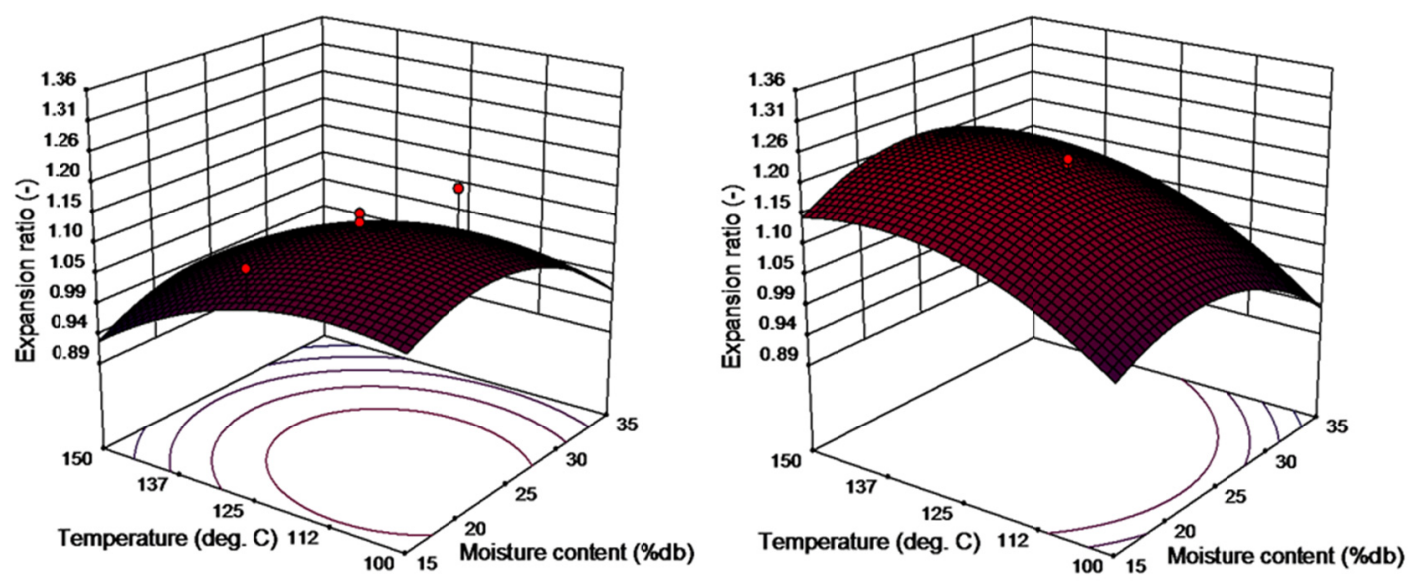

(A)

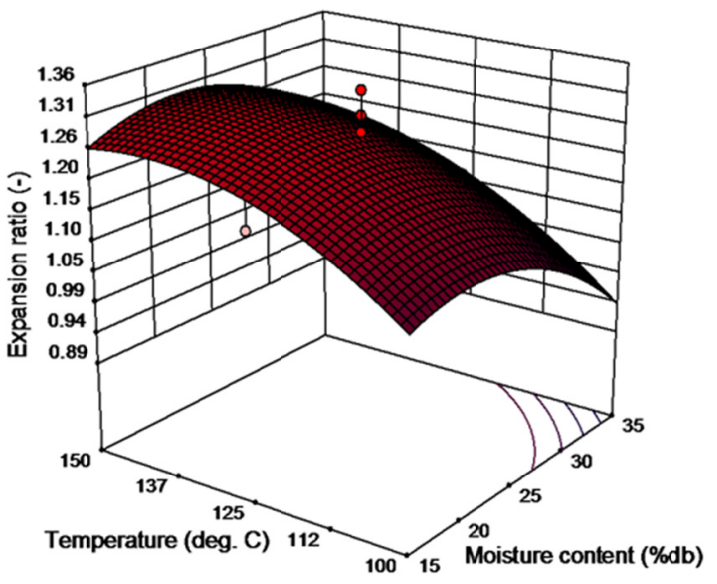

(B)

(C)

Figure 5. Response surface plots of expansion ratio for the effect of temperature and moisture content at $40 \% \mathrm{db}$ soy white flakes at different die aspect ratio (L/D), (A) 3.33, (B) 5.83, and (C) 7.25.

The experimental values of expansion ratio of extrudates under different designed extrusion conditions are shown in Table 9. The maximum and minimum expansion ratio were achieved at $52 \%$ soy white flakes, $19 \%$ moisture content, $140{ }^{\circ} \mathrm{C}$, and $7.25 \mathrm{~L} / \mathrm{D}$ ratio and $40 \%$ soy white flakes, $25 \%$ moisture content, $150{ }^{\circ} \mathrm{C}$ temperature, and $3.33 \mathrm{~L} / \mathrm{D}$ ratio, respectively 
Table 9a. Treatment combination effects for soy white flakes, moisture content of raw material, temperature and die on extrudate physical properties

\begin{tabular}{|c|c|c|c|c|c|}
\hline Treatment & $\begin{array}{l}\text { PDI } \\
(\%)\end{array}$ & $\begin{array}{l}\text { BD } \\
(g / c c)\end{array}$ & $\begin{array}{l}\text { WAI } \\
\text { (unitless) }\end{array}$ & $\begin{array}{l}\text { WSI } \\
(\%)\end{array}$ & $\begin{array}{l}\text { ER } \\
\text { (unitless) }\end{array}$ \\
\hline 1 & $90.09 \pm 0.18^{16-18}$ & $0.46 \pm 0.01^{12-14}$ & $3.62 \pm 0.05^{23-25}$ & $14.97 \pm 0.11^{4-8}$ & $1.06 \pm 0.02^{27-29}$ \\
\hline 2 & $87.49 \pm 0.02^{23}$ & $0.39 \pm 0.00^{21-22}$ & $4.70 \pm 0.11^{5}$ & $13.50 \pm 0.39^{18-23}$ & $1.22 \pm 0.01^{7-11}$ \\
\hline 3 & $85.30 \pm 0.10^{26-27}$ & $0.49 \pm 0.01^{4-6}$ & $3.72 \pm 0.10^{21-25}$ & $14.83 \pm 0.41^{4-11}$ & $1.06 \pm 0.01^{27-29}$ \\
\hline 4 & $85.93 \pm 0.79^{25}$ & $0.47 \pm 0.01^{9-11}$ & $5.14 \pm 0.11^{3-4}$ & $13.56 \pm 0.20^{17-22}$ & $1.12 \pm 0.01^{18-23}$ \\
\hline 5 & $97.03 \pm 0.13^{2-3}$ & $0.48 \pm 0.01^{6-9}$ & $4.34 \pm 0.12^{7-9}$ & $14.48 \pm 0.25^{7-14}$ & $1.131 \pm 0.00^{8-22}$ \\
\hline 6 & $89.75 \pm 0.28^{17-18}$ & $0.48 \pm 0.00^{8-10}$ & $4.81 \pm 0.10^{5}$ & $13.15 \pm 0.21^{22-24}$ & $1.09 \pm 0.02^{23-27}$ \\
\hline 7 & $95.61 \pm 0.47^{4-5}$ & $0.44 \pm 0.01^{14-16}$ & $4.64 \pm 0.14^{5-6}$ & $14.29 \pm 0.40^{10-16}$ & $1.07 \pm 0.02^{26-28}$ \\
\hline 8 & $92.01 \pm 0.16^{14}$ & $0.49 \pm 0.01^{4-5}$ & $5.39 \pm 0.06^{2}$ & $13.31 \pm 0.18^{21-24}$ & $1.16 \pm 0.02^{15-18}$ \\
\hline 9 & $90.94 \pm 0.14^{15}$ & $0.33 \pm 0.00^{25}$ & $4.15 \pm 0.12^{10-15}$ & $13.85 \pm 0.38^{15-21}$ & $1.32 \pm 0.01^{2}$ \\
\hline 10 & $88.00 \pm 0.30^{22-23}$ & $0.50 \pm 0.02^{4}$ & $4.23 \pm 0.07^{8-12}$ & $14.43 \pm 0.18^{7-16}$ & $1.03 \pm 0.01^{30-31}$ \\
\hline 11 & $90.51 \pm 0.63^{15-16}$ & $0.45 \pm 0.01^{13-15}$ & $3.54 \pm 0.14^{25-26}$ & $14.88 \pm 0.32^{4-10}$ & $1.08 \pm 0.02^{26-28}$ \\
\hline 12 & $92.36 \pm 0.11^{13-14}$ & $0.49 \pm 0.01^{4-7}$ & $5.16 \pm 0.08^{3-4}$ & $13.40 \pm 0.32^{19-24}$ & $0.96 \pm 0.01^{32}$ \\
\hline 13 & $81.75 \pm 0.15^{30}$ & $0.33 \pm 0.01^{25}$ & $5.30 \pm 0.05^{2-3}$ & $12.83 \pm 0.23^{24}$ & $1.29 \pm 0.02^{2-3}$ \\
\hline 14 & $90.80 \pm 0.17^{15}$ & $0.38 \pm 0.01^{22}$ & $4.29 \pm 0.14^{7-11}$ & $13.83 \pm 0.26^{16-21}$ & $1.10 \pm 0.01^{21-26}$ \\
\hline 15 & $92.01 \pm 0.21^{14}$ & $0.34 \pm 0.01^{24-25}$ & $5.04 \pm 0.14^{4}$ & $12.94 \pm 0.34^{23-24}$ & $1.28 \pm 0.02^{3-5}$ \\
\hline 16 & $90.21 \pm 0.22^{16-17}$ & $0.34 \pm 0.01^{24-25}$ & $5.59 \pm 0.05^{1}$ & $12.91 \pm 0.11^{23-24}$ & $1.41 \pm 0.02^{1}$ \\
\hline 17 & $90.17 \pm 0.42^{16-17}$ & $0.47 \pm 0.00^{8-11}$ & $3.71 \pm 0.08^{22-25}$ & $14.57 \pm 0.25^{7-13}$ & $1.05 \pm 0.00^{28-31}$ \\
\hline 18 & $93.13 \pm 0.25^{10-12}$ & $0.36 \pm 0.01^{23}$ & $4.47 \pm 0.01^{6-7}$ & $13.262 \pm 0.25^{1-24}$ & $1.28 \pm 0.02^{3-4}$ \\
\hline 19 & $84.29 \pm 0.41^{28}$ & $0.46 \pm 0.00^{11-13}$ & $3.10 \pm 0.13^{28-29}$ & $15.19 \pm 0.36^{3-6}$ & $1.08 \pm 0.00^{25-27}$ \\
\hline 20 & $97.54 \pm 0.19^{2}$ & $0.47 \pm 0.01^{10-12}$ & $3.15 \pm 0.07^{28-29}$ & $15.27 \pm 0.28^{3-5}$ & $1.15 \pm 0.01^{16-19}$ \\
\hline 21 & $90.16 \pm 0.22^{16-17}$ & $0.50 \pm 0.01^{4}$ & $3.93 \pm 0.14^{16-20}$ & $14.25 \pm 0.18^{11-16}$ & $1.10 \pm 0.01^{21-26}$ \\
\hline 22 & $96.71 \pm 0.14^{3}$ & $0.49 \pm 0.00^{4-6}$ & $3.74 \pm 0.07^{20-23}$ & $14.99 \pm 0.07^{4-7}$ & $1.14 \pm 0.01^{17-20}$ \\
\hline 23 & $98.11 \pm 0.37^{1}$ & $0.44 \pm 0.01^{14-16}$ & $3.37 \pm 0.09^{26-27}$ & $15.01 \pm 0.08^{4-7}$ & $1.13 \pm 0.01^{18-21}$ \\
\hline 24 & $88.91 \pm 0.38^{20-21}$ & $0.54 \pm 0.00^{2}$ & $3.03 \pm 0.02^{29}$ & $15.70 \pm 0.12^{2-3}$ & $1.09 \pm 0.01^{23-27}$ \\
\hline 25 & $92.25 \pm 0.18^{14}$ & $0.44 \pm 0.00^{16-18}$ & $4.04 \pm 0.12^{13-18}$ & $15.77 \pm 0.33^{1-3}$ & $1.19 \pm 0.02^{11-14}$ \\
\hline 26 & $93.29 \pm 0.05^{10-12}$ & $0.38 \pm 0.01^{21-22}$ & $4.12 \pm 0.05^{11-15}$ & $14.15 \pm 0.25^{12-17}$ & $1.25 \pm 0.01^{4-7}$ \\
\hline 27 & $94.84 \pm 0.13^{6}$ & $0.52 \pm 0.00^{3}$ & $4.03 \pm 0.09^{14-18}$ & $14.46 \pm 0.12^{7-15}$ & $1.02 \pm 0.01^{31}$ \\
\hline 28 & $92.87 \pm 0.31^{11-13}$ & $0.38 \pm 0.00^{22}$ & $4.34 \pm 0.15^{7-10}$ & $14.35 \pm 0.18^{9-16}$ & $1.23 \pm 0.02^{6-9}$ \\
\hline 29 & $83.23 \pm 0.18^{29}$ & $0.41 \pm 0.01^{19-20}$ & $3.07 \pm 0.09^{29}$ & $16.26 \pm 0.18^{1-2}$ & $1.19 \pm 0.02^{11-14}$ \\
\hline 30 & $89.92 \pm 0.36^{17-18}$ & $0.43 \pm 0.01^{18}$ & $4.11 \pm 0.12^{11-16}$ & $14.04 \pm 0.35^{13-18}$ & $1.19 \pm 0.01^{12-15}$ \\
\hline
\end{tabular}

+ The values with the same superscript for a given property are not significantly different $(\mathrm{P}<0.05)$. PDI - Pellet durability index, BD - Bulk density, WAI - Water absorption index, WSI - Water solubility index, ER Expansion ratio. 
Table $9 \mathrm{~b}$. Treatment combination effects for soy white flakes, moisture content of raw material, temperature and die on extrudate physical properties

\begin{tabular}{|c|c|c|c|c|c|}
\hline Treatment & $\begin{array}{l}\text { PDI } \\
(\%)\end{array}$ & $\begin{array}{l}\text { BD } \\
(g / c c)\end{array}$ & $\begin{array}{l}\text { WAI } \\
\text { (unitless) }\end{array}$ & $\begin{array}{l}\text { WSI } \\
(\%)\end{array}$ & $\begin{array}{l}\text { ER } \\
\text { (unitless) }\end{array}$ \\
\hline 31 & $85.77 \pm 0.18^{25-26}$ & $0.43 \pm 0.01^{18}$ & $3.91 \pm 0.10^{17-21}$ & $14.34 \pm 0.16^{9-16}$ & $1.23 \pm 0.02^{6-10}$ \\
\hline 32 & $85.12 \pm 0.43^{27}$ & $0.40 \pm 0.01^{20-21}$ & $4.15 \pm 0.14^{10-15}$ & $14.31 \pm 0.33^{9-16}$ & $1.21 \pm 0.02^{8-12}$ \\
\hline 33 & $93.55 \pm 0.07^{9-10}$ & $0.53 \pm 0.01^{2}$ & $3.36 \pm 0.08^{26-27}$ & $15.68 \pm 0.07^{2-3}$ & $1.09 \pm 0.01^{23-27}$ \\
\hline 34 & $92.81 \pm 0.50^{12-13}$ & $0.35 \pm 0.01^{24}$ & $4.03 \pm 0.04^{14-18}$ & $14.34 \pm 0.16^{9-16}$ & $1.24 \pm 0.01^{5-8}$ \\
\hline 35 & $96.72 \pm 0.47^{3}$ & $0.44 \pm 0.01^{15-17}$ & $4.16 \pm 0.08^{9-14}$ & $14.37 \pm 0.40^{8-16}$ & $1.12 \pm 0.02^{19-24}$ \\
\hline 36 & $96.03 \pm 0.11^{4}$ & $0.46 \pm 0.00^{11-13}$ & $3.42 \pm 0.01^{26-27}$ & $15.79 \pm 0.21^{1-3}$ & $1.09 \pm 0.01^{22-27}$ \\
\hline 37 & $86.19 \pm 0.96^{24-25}$ & $0.47 \pm 0.01^{10-12}$ & $4.36 \pm 0.19^{7-8}$ & $13.84 \pm 0.22^{16-21}$ & $1.09 \pm 0.01^{23-27}$ \\
\hline 38 & $94.59 \pm 0.35^{6-7}$ & $0.57 \pm 0.01^{1}$ & $3.43 \pm 0.07^{26-27}$ & $15.35 \pm 0.25^{3-4}$ & $1.12 \pm 0.01^{18-23}$ \\
\hline 39 & $88.43 \pm 0.06^{21-22}$ & $0.48 \pm 0.02^{5-8}$ & $3.90 \pm 0.06^{17-22}$ & $14.35 \pm 0.17^{9-16}$ & $1.12 \pm 0.01^{19-25}$ \\
\hline 40 & $92.02 \pm 0.16^{14}$ & $0.47 \pm 0.00^{9-11}$ & $4.07 \pm 0.04^{12-18}$ & $13.34 \pm 0.35^{20-24}$ & $1.03 \pm 0.01^{29-31}$ \\
\hline 41 & $93.06 \pm 0.18^{10-12}$ & $0.45 \pm 0.01^{14-16}$ & $3.55 \pm 0.06^{24-26}$ & $14.73 \pm 0.27^{5-12}$ & $1.16 \pm 0.00^{15-18}$ \\
\hline 42 & $93.91 \pm 0.29^{8-9}$ & $0.37 \pm 0.00^{23}$ & $4.08 \pm 0.15^{12-17}$ & $14.41 \pm 0.18^{7-16}$ & $1.12 \pm 0.01^{20-25}$ \\
\hline 43 & $93.50 \pm 0.09^{9-10}$ & $0.32 \pm 0.01^{26}$ & $4.11 \pm 0.17^{11-16}$ & $15.34 \pm 0.23^{3-5}$ & $1.26 \pm 0.01^{3-6}$ \\
\hline 44 & $90.18 \pm 0.20^{16-17}$ & $0.40 \pm 0.00^{20-21}$ & $3.38 \pm 0.02^{26-27}$ & $15.62 \pm 0.44^{3}$ & $1.17 \pm 0.00^{14-17}$ \\
\hline 45 & $87.75 \pm 0.47^{23}$ & $0.41 \pm 0.01^{19}$ & $4.05 \pm 0.14^{12-18}$ & $14.05 \pm 0.28^{13-18}$ & $1.19 \pm 0.02^{10-14}$ \\
\hline 46 & $89.81 \pm 0.25^{17-18}$ & $0.43 \pm 0.01^{17-18}$ & $3.97 \pm 0.03^{15-19}$ & $14.13 \pm 0.19^{12-17}$ & $1.18 \pm 0.02^{13-17}$ \\
\hline 47 & $83.37 \pm 0.66^{29}$ & $0.47 \pm 0.00^{8-11}$ & $3.63 \pm 0.05^{23-25}$ & $14.59 \pm 0.21^{6-13}$ & $1.13 \pm 0.01^{18-22}$ \\
\hline 48 & $92.09 \pm 0.06^{14}$ & $0.39 \pm 0.01^{21-22}$ & $4.46 \pm 0.04^{6-7}$ & $14.01 \pm 0.34^{13-18}$ & $1.17 \pm 0.01^{13-17}$ \\
\hline 49 & $89.61 \pm 0.25^{18-19}$ & $0.48 \pm 0.01^{7-10}$ & $3.53 \pm 0.03^{25-26}$ & $14.48 \pm 0.14^{7-14}$ & $1.08 \pm 0.02^{26-28}$ \\
\hline 50 & $94.88 \pm 0.57^{6}$ & $0.44 \pm 0.01^{16-18}$ & $3.39 \pm 0.06^{26-27}$ & $15.35 \pm 0.43^{3-4}$ & $1.06 \pm 0.01^{27-30}$ \\
\hline 51 & $94.58 \pm 0.10^{6-7}$ & $0.40 \pm 0.01^{19-20}$ & $3.01 \pm 0.08^{29}$ & $16.26 \pm 0.21^{1-2}$ & $1.17 \pm 0.02^{13-17}$ \\
\hline 52 & $86.59 \pm 0.27^{24}$ & $0.33 \pm 0.01^{25}$ & $3.89 \pm 0.04^{18-22}$ & $14.57 \pm 0.21^{7-13}$ & $1.28 \pm 0.02^{3-4}$ \\
\hline 53 & $93.40 \pm 0.22^{9-11}$ & $0.44 \pm 0.00^{15-16}$ & $4.39 \pm 0.01^{7-8}$ & $13.98 \pm 0.26^{13-19}$ & $1.03 \pm 0.01^{29-31}$ \\
\hline 54 & $97.46 \pm 0.18^{2}$ & $0.44 \pm 0.00^{14-16}$ & $3.26 \pm 0.04^{27-28}$ & $16.33 \pm 0.38^{1}$ & $1.18 \pm 0.01^{13-17}$ \\
\hline 55 & $95.11 \pm 0.31^{5-6}$ & $0.33 \pm 0.01^{25}$ & $5.02 \pm 0.03^{4}$ & $14.36 \pm 0.35^{8-16}$ & $1.18 \pm 0.02^{12-16}$ \\
\hline 56 & $88.81 \pm 0.24^{20-21}$ & $0.46 \pm 0.00^{12-14}$ & $3.92 \pm 0.04^{17-20}$ & $14.41 \pm 0.30^{7-16}$ & $1.09 \pm 0.02^{24-27}$ \\
\hline 57 & $98.37 \pm 0.61^{1}$ & $0.44 \pm 0.00^{14-16}$ & $3.74 \pm 0.02^{20-24}$ & $15.32 \pm 0.21^{3-5}$ & $1.20 \pm 0.01^{9-13}$ \\
\hline 58 & $94.24 \pm 0.19^{7-8}$ & $0.34 \pm 0.01^{25}$ & $3.78 \pm 0.02^{19-23}$ & $14.92 \pm 0.37^{4-9}$ & $1.24 \pm 0.01^{6-9}$ \\
\hline 59 & $85.88 \pm 0.22^{25}$ & $0.32 \pm 0.00^{26}$ & $4.23 \pm 0.09^{8-13}$ & $14.22 \pm 0.07^{12-16}$ & $1.25 \pm 0.02^{4-7}$ \\
\hline 60 & $89.17 \pm 0.43^{19-20}$ & $0.48 \pm 0.00^{8-10}$ & $4.37 \pm 0.04^{7-8}$ & $13.95 \pm 0.18^{14-20}$ & $0.90 \pm 0.01^{33}$ \\
\hline
\end{tabular}

+ The values with the same superscript for a given property are not significantly different $(\mathrm{P}<0.05)$. PDI - Pellet durability index, BD - Bulk density, WAI - Water absorption index, WSI - Water solubility index, ER Expansion ratio.

\section{Conclusions}

This experimental study was conducted to investigate the effect of various extrusion processing conditions on the soy white flakes and HP-DDG based extrudates. Overall, it can be concluded that increasing the level of soy white flakes from $21 \%$ to $59 \%$, resulted in increase of pellet durability and water absorption index. Increasing L/D ratio from 3.33 to 7.25 resulted in increase in pellet durability index, expansion ratio, but a decrease in bulk density of the extrudates. The increase in pellet durability indicates that the aquaculture feed could resist 
mechanical damage during transportation and storage. Significant decrease in bulk density $(P<0.001)$ due to increase in $\mathrm{L} / \mathrm{D}$ ratio is desirable for storage purpose. Further studies should aim for the production of aquaculture feed with incorporation of soy white flakes levels between $20 \%$ and $60 \% \mathrm{db}$ at different screw speeds and should optimize processing conditions.

\section{Acknowledgements}

The authors thank the USDA-North Central Agricultural Research Laboratory, Brookings, South Dakota, Agricultural Experiment Station, South Dakota State University, and Indian Council of Agricultural Research (ICAR), New Delhi, India for funding, facilities, equipment and supplies.

\section{References}

AACC. (2000). Method 44-19, moisture-air oven method, drying at $135{ }^{\circ} \mathrm{C}$. AACC Approved Methods, (10th Ed.) American Association of Cereal Chemists: St. Paul, MN.

Alves, R. M. L., Grossmann, M. V. E., \& Silva, R. S. S. F. (1999). Gelling properties of extruded yam (Dioscorea alota) starch. Food Chemistry, 67, 123-127. http://dx.doi.org/10.1016/S0308-8146(99)00064-3

Anderson, R. A., Conway, H. F., Pfeifer, V. F., \& Griffin, E. L. (1969). Roll and extrusion cooking of grain sorghum grits. Cereal Sci. Today, 14(11), 373-375.

Anderson, R. A., Conway, H. F., Pfeifer, V. F., \& Griffin, E.L. (1969). Gelatinization of corn grits by roll and extrusion cooking. Cereal Science Today, 14, 4-7.

ASAE. (2004). Engineering Standards, Practices and Data. ASABE, St. Joseph, MI.

Ayadi, F. Y., Fallahi, P., Rosentrater, K. A., \& Muthukumarappan, K. (2013). Modeling single-screw extrusion processing parameters and resulting extrudate properties of DDGS-based Nile Tilapia (Oreochromis niloticus) Feeds. Journal of Food Research, 2(2). http://dx.doi.org/10.5539/jfr.v2n2p11

Bandyopadhyay, S., \& Ranjan, K. R. (2001). Aquafeed extrudate flow rate and pellet characteristics from low cost single screw extruder. Journal of Aquatic Food Product Technology, 10(2), 3-14. http://dx.doi.org/10.1300/J030v10n02_02

Bhattacharya, M., \& Hanna, M. A. (1986). Viscosity modeling of dough in extrusion. J. Food Process Eng, 2, 337-342.

Case, S. E., Hamann, D. D., \& Schwartz, S. J. (1992). Effect of starch gelatinization on physical properties of extruded wheat and corn based products. Cereal Chem, 69(4), 401-404.

Chevanan, N., Muthukumarappan, K., Rosentrater, K. A., \& Julson, J. L. (2007). Effect of die dimensions on extrusion processing parameters and properties of DDGS-based aquaculture feeds. Cereal chemistry, 84(4), 389-398.

Chin, H. K., Joseph, A. M., \& Jeffery, T. M. (1989). Properties of extruded dried distillers grains (DDG) and flour blends. J. Food processing and preservation, 13, 219-231. http://dx.doi.org/10.1111/j.1745-4549.1989.tb00102.x

Chinnaswamy, R., \& Hanna, M. A. (1987). Nozzle dimension effects on the expansion of extrusion cooked corn starch. J. Food Sci, 52(6), 1746-1747. http://dx.doi.org/10.1111/j.1365-2621.1987.tb05924.x

Dersjant-Li, Y. (2002). The use of soy protein in aquafeeds. In The use of soy protein in aquafeeds. In Cruz-suárez, l. E., ricque-marie, d., tapia-salazar, m., gaxiola-cortés, m. G., simoes, n. (Eds.). Avances en nutriciónacuícolavi. Memorias del vi symposium 76 internacional de nutriciónacuícola. 3 al 6 deseptiembredel 2002. Cancún, quintanaroo, méxico.

El-Sayed, A.-F. M. (1999). Alternative protein sources for farmed tilapia, Oreochromis spp. Aquaculture, 179, 149-168. http://dx.doi.org/10.1016/S0044-8486(99)00159-3

El-Sayed, A.-F. M. (2004). Protein nutrition of farmed tilapia: searching for unconventional sources. In R. Bolivar, G. Mair \& K. Fitzsimmons (Eds.) (pp. 364-378) Sixth International Symposium on Tilapia in Aquaculture, Manila, Philippines.

Fallahi, P., Muthukumarappan, K., \& Rosentrater, K. A. (2012). Twin-screw Extrusion Processing of Vegetable-based Protein Feeds for Yellow Perch (Percaflavescens) Containing Distillers Dried Grains, Soy Protein Concentrate, and Fermented High Protein Soybean Meal. Journal of Food Research, 1(3), 230-246. http://dx.doi.org/10.5539/jfr.v1n3p230

Fallahi, P., Rosentrater, K. A., Muthukumarappan, K., \& Tulbek, M. (2013). Effects of Steam, Moisture, and 
Screw Speed on Physical Properties of DDGS-Based Extrudates. Cereal Chem, 90(3), 186-197. http://dx.doi.org/10.1094/CCHEM-08-12-0102-R

FAO. (2008). The state of the world fisheries aquaculture. Food and Agriculture Organization, Rome, Italy.

FAO. (2012). The state of the world fisheries aquaculture. Food and Agriculture Organization, Rome, Italy.

Gwiazda, S., Noguchi, A., \& Saio, K. (1987). Microstructural studies of texturized vegetable protein products: Effects of oil addition and transformation of raw materials in various sections of a twin screw extruder. Food Microstructure, 6, 57-61.

Hardy, R. W. (2010). Utilization of plant proteins in fish diets: Effects of global demand and supplies of fishmeal. Aquaculture Research, 41, 770-776. http://dx.doi.org/10.1111/j.1365-2109.2009.02349.x

Harper, J. M, Rhodes, T. P., \& Wanninger, L. A. (1971). Viscosity model for cooked cereal dough. AIChESymp. Ser, 67(108), 40-43.

Ibanoglu, S., Paul, A., \& Geroge, D. H. (1996). Extrusion of tarhana: effect of operating variables on starch gelatinization. Food Chemistry, 57(4), 541-544. http://dx.doi.org/10.1016/S0308-8146(96)00047-7

Ilo, S., Tomschik, E. U., Berghofer., \& Mundigler, N. (1996). The effects of extrusion operating conditions on the apparent viscosity and properties of extrudates in twin screw extrusion cooking of maize grits. Lebensm.-Wiss.u.-Technol, 29, 593-598.

Kannadhason, S., Rosentrater, K. A., \& Muthukumarappan, K. (2010). Twin screw extrusion of DDGS-based aquaculture feeds. Journal of the World Aquaculture Society, 41(51), 1-15. http://dx.doi.org/10.1111/j.1749-7345.2009.00328.x

Kokini, L. J., Ho, C. T., \& Karwe, M. V. (1992a). Food extrusion science and technology. Marcel Dekker, Inc.: New York.

Kokini, J. L, Chang, C. N., \& Lai, L. S. (1992b). The role of rheological properties of extrudate expansion. In Kokini, J. L., Ho, C. T., \& Karwe, M., (Eds.), Food extrusion science and Technology (pp. 631-652). Marcel Dekker, Inc. New York.

Lam, C. D., \& Rolando, A. F. (2003). Effect of particle size and moisture content on viscosity of fish feed. Cereal Chem, 80(1), 20-24. http://dx.doi.org/10.1094/CCHEM.2003.80.1.20

Lin, S., Huff, H. E., \& Hsien, F. (2000). Texture and chemical characteristics of soy protein meat analog $\begin{array}{lllll}\text { extruded at high moisture. J. Food } & \text { Sci, 65(2), } & \text { 264-269. }\end{array}$ http://dx.doi.org/10.1111/j.1365-2621.2000.tb15991.x

Lovell, T. (1988). Nutrition and feeding of fish. Van Nostrand Reinhold: New York.

Mason, W. R., \& Hoseney, R. C. (1986). Factors affecting the viscosity of extrusion-cooked wheat starch. Cereal Chem, 63, 436-441.

Mercier, C., Linko, P., \& Harper, J. M. (1989). Extrusion cooking. American Association of Cereal Chemists, Inc., St. Paul, Minnesota.

Rolfe, L. A., Huff, H. E., \& Hsieh, F. (2001). Effects of particle size and processing variables on the properties of an extruded catfish feed. Journal of aquatic food product technology, 10(3), 21-33. http://dx.doi.org/10.1300/J030v10n03_03

Romarheim, O. H., Aslaksen, M. A., Storebakken, T., Krogdahl, A., \& Skrede, A. (2005). Effect of extrusion on trypsin inhibitor activity and nutrient digestibility of diets based on fish meal, soybean meal and white flakes. Archives of Animal Nutrition, 59(6), 365-375. http://dx.doi.org/10.1080/17450390500352897

Rosentrater, K. A., Richard, T. L., Bern, C. J., \& Flores, R. A. (2005). Small-scale extrusion of corn masa by-products. Cereal Chemistry, 82(4), 436-446. http://dx.doi.org/10.1094/CC-82-0436

Sandra, H. P. F., \& Jose, A. G. A. (1993). Effect of phospholipid on protein structure and solubility in the extrusion of lung proteins. Food Chemistry, 47, 111-119. http://dx.doi.org/10.1016/0308-8146(93)90230-D

Shukla, C. Y., Muthukumarappan, K., \& Julson, J. L. (2005). Effect of single screw extruder die temperature, amount of distiller dried grains with solubles (DDGS) and initial moisture content on extrudates. Cereal Chem, 82(1), 34-37. http://dx.doi.org/10.1094/CC-82-0034

Singh, R. K., Nielsen, S. S., \& Chambers, J. V. (1991). Selected characteristics of extruded blends of milk protein raffinate or nonfat dry milk with corn flour. Journal of Food processing and Preservation, 15, 
285-302. http://dx.doi.org/10.1111/j.1745-4549.1991.tb00173.x

Sokhey, A. S., Kollengode, A. N., \& Hanna, M. A. (1994). Screw configuration effects on corn starch expansion during extrusion. J. Food Sci, 59(4), 895-899. http://dx.doi.org/10.1111/j.1365-2621.1994.tb08152.x

USDA. (2009). Practical Procedures for Grain Handlers: Inspecting Grain. United States Department of Agriculture - Grain Inspection, Packers, and Stockyards Administration: Washington, D.C. Retrieved from http://www.gipsa.usda.gov/publications/fgis/ref/primer.pdf

Williams, M. A., Horn, R. E., \& Rugula, R. P. (1977). Extrusion: An in depth look at a versatile process. I. J. Food Eng., 49(10), 87-89.

Wu, Y. V., Ronald, R. R., David, J. S., \& Paul, B. B. (1994). Utilization of protein rich Ethanol co-products from Corn in tilapia feed. JAOCS, 71(9), 1041-1043. http://dx.doi.org/10.1007/BF02542277

Wu, Y. V., Ronald, R. R., David, J. S., \& Paul, B. B. (1996a). Effects of diets containing various levels of protein and ethanol coproducts from corn on growth of tilapia fry. J. Agric. Food Chem, 440, 1491-1. http://dx.doi.org/10.1021/j9950733g

\section{Copyrights}

Copyright for this article is retained by the author(s), with first publication rights granted to the journal.

This is an open-access article distributed under the terms and conditions of the Creative Commons Attribution license (http://creativecommons.org/licenses/by/3.0/). 